\title{
Multicentric Castleman's disease as a cause for unclear febrile episodes in a 55-year-old HIV-infected man
}

\author{
Lederer, H ; Achermann, Y ; Tinguely, M ; Stenner, F ; Fehr, J
}

\begin{abstract}
Our case illustrates the difficulties involved in diagnosing multicentric Castleman's disease (MCD) in a human immunodeficiency virus-infected man with febrile episodes and malaise. In the absence of well-established treatment protocols, we have chosen a new treatment algorithm with rituximab, etoposide, and valganciclovir, which led to the remission of clinical symptoms. Yet, we advocate focused exploration for MCD in immunosuppressed patients with unclear febrile episodes, as recent advances in treatment are promising.
\end{abstract}

DOI: https://doi.org/10.1007/s15010-011-0174-9

Posted at the Zurich Open Repository and Archive, University of Zurich

ZORA URL: https://doi.org/10.5167/uzh-55895

Journal Article

Published Version

Originally published at:

Lederer, H; Achermann, Y; Tinguely, M; Stenner, F; Fehr, J (2012). Multicentric Castleman's disease as a cause for unclear febrile episodes in a 55-year-old HIV-infected man. Infection, 40(2):203-205.

DOI: https://doi.org/10.1007/s15010-011-0174-9 


\title{
Multicentric Castleman's disease as a cause for unclear febrile episodes in a 55-year-old HIV-infected man
}

\author{
H. Lederer $\cdot$ Y. Achermann $\cdot$ M. Tinguely $\cdot$ \\ F. Stenner $\cdot$ J. Fehr
}

Received: 3 April 2011/Accepted: 27 July 2011/Published online: 12 August 2011

(C) Springer-Verlag 2011

\begin{abstract}
Our case illustrates the difficulties involved in diagnosing multicentric Castleman's disease (MCD) in a human immunodeficiency virus-infected man with febrile episodes and malaise. In the absence of well-established treatment protocols, we have chosen a new treatment algorithm with rituximab, etoposide, and valganciclovir, which led to the remission of clinical symptoms. Yet, we advocate focused exploration for MCD in immunosuppressed patients with unclear febrile episodes, as recent advances in treatment are promising.
\end{abstract}

Keywords Febrile episodes · Immunosuppression · Multicentric Castleman's disease

H. Lederer

Department of Internal Medicine,

University Hospital Zurich,

University of Zurich, Zurich, Switzerland

Y. Achermann $\cdot$ J. Fehr $(\varangle)$

Division of Infectious Diseases and Hospital Epidemiology,

University Hospital Zurich, University of Zurich,

Rämistrasse 100, 8091 Zurich, Switzerland

e-mail: jan.fehr@usz.ch

M. Tinguely

Department of Pathology,

University Hospital Zurich,

University of Zurich, Zurich, Switzerland

F. Stenner

Department of Oncology, University Hospital Zurich,

University of Zurich, Zurich, Switzerland

\section{Introduction}

In 1954, Benjamin Castleman first described a series of cases which was regarded as a non-neoplastic polyclonal lymphoproliferative disease or 'pre-lymphoma' [1]. Initially, it has been classified based upon clinical presentation (uni- vs. multicentric) and morphology (hyaline vascular type vs. plasma cell type). In the setting of immunosuppressed, particularly, human immunodeficiency virus (HIV)-infected individuals, there is an increased recognition of the human herpes virus 8 (HHV-8)-associated form with variable morphology, clinically presenting as multicentric Castleman's disease (MCD). Despite mostly behaving as non-malignant disease, its influence on morbidity and mortality is significant [2].

\section{Case description}

In February 2010, a 55-year-old homosexual man with HIV infection was referred to our hospital with symptoms of fever, weakness, and diarrhea. For the past 3 months, he has been suffering from several febrile episodes lasting 2-5 days with spontaneous resolution. Extensive work-up during previous hospitalizations failed to reveal a cause.

HIV has been diagnosed in 2007 and the patient was treated with an antiretroviral regimen, including lopinavir/ ritonavir, lamivudine, and efavirenz. Despite excellent virological response with undetectable plasma viral load and several modifications in his antiretroviral medication, the patient remained severely immunosuppressed with a maximum of $107 \mathrm{CD} 4^{+}$T-lymphocytes/ $\mu \mathrm{l}(5 \%)$.

On admission, our patient was in poor general condition, febrile $\left(39.5^{\circ} \mathrm{C}\right)$ with a regular heart rate $(74 / \mathrm{min})$, hypotensive $(95 / 54 \mathrm{mmHg}$ ), and presented multiple, slightly 
Fig. 1 Lymph node histology showing: a a lymph follicle with a regressed germinal center and radially penetrating blood vessels (right), accompanied by an increased interfollicular plasma cell content (asterisk); b double-staining for the light chains kappa (brown) and lambda (blue) highlights the massive increase of polytypic plasma cells; c nuclear staining for the latency-associated nuclear antigen (LANA-1) in a few plasmablasts within the follicular mantle zone

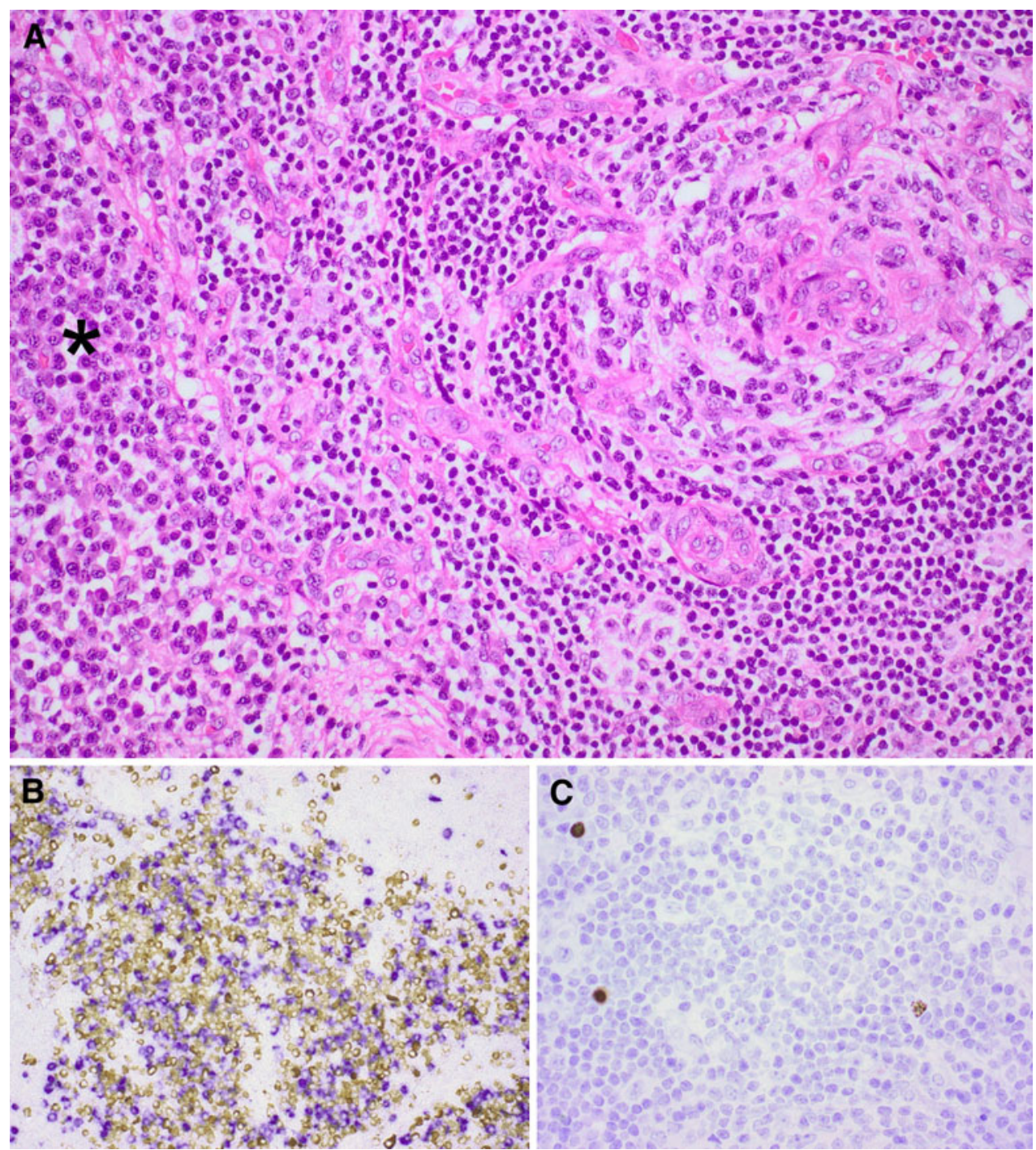

enlarged cervical and inguinal lymph nodes and an enlarged spleen. Laboratory tests showed pancytopenia (hemoglobin of $7.4 \mathrm{~g} / \mathrm{dl}$, neutrophils $1.26 \times 10^{3} / \mu \mathrm{l}$, platelets $103 \times 10^{3} /$ $\mu \mathrm{l}$ ), an elevated C-reactive protein (CRP, $228 \mathrm{mg} / \mathrm{l}$, normal range $<5 \mathrm{mg} / \mathrm{l}$ ), and acute renal failure (creatinine $315 \mu \mathrm{mol} / \mathrm{l}$ ). Several sets of blood cultures, examinations of stool for bacteria, helminths, protozoas, and corresponding serology remained negative. Mycobacterial infection was excluded by repeated blood, respiratory, stool, and urine samples. Upper and lower endoscopies were performed and tissue biopsies showed no evidence of Tropheryma whipp$l e i$, neoplasia, or inflammatory bowel disease. Bone marrow aspiration and biopsy demonstrated non-specific changes without dysplastic or myeloproliferative changes and polymerase chain reaction (PCR) for leishmaniasis and mycobacteriosis was negative. No evidence for hemophagocytotic syndrome was found.

Thoraco-abdominal computed tomography (CT) confirmed splenomegaly (maximal longitudinal diameter
$21 \mathrm{~cm}$ ) and slightly enlarged iliac and hilar lymph nodes. Fluorodeoxyglucose (FDG) positron emission tomography scan revealed a pathological FDG uptake in axillary, cervical and supraclavicular lymph nodes, spleen, and bone marrow. Histological examination of a cervical lymph node showed distorted secondary follicles with regressed germinal centers, hyperplastic follicular dendritic cells, and a significant surplus of interfollicular plasma cells.

Immunohistochemical staining was positive for HHV-8 latency-associated nuclear antigen (LANA) (Fig. 1). The patient's plasma HHV-8 viral load was 31,111 copies $/ \mathrm{ml}$. The diagnosis of MCD could be made based on these findings.

Treatment with rituximab, etoposide, and valganciclovir for six cycles according to the algorithm recently proposed by Oksenhendler [2] was started. One year later, our patient is still free from new episodes of fever and is working fulltime. Follow-up CT scan revealed a decreased size and number of lymph nodes and spleen (longitudinal diameter $17 \mathrm{~cm}$ ). Plasma HHV-8 viral load is no longer detectable. 


\section{Discussion}

Disease presentation in MCD varies widely and is nonspecific. The most common signs and symptoms include malaise, fever, night sweats, weight loss, generalized lymphadenopathy, splenomegaly, edema, and cytopenia [3, 4]. Untreated MCD exhibits a progressive clinical course which may result in malignancy and death. To confirm the diagnosis, the detection of HHV-8-infected cells using immunohistochemical techniques in lymph nodes or bone marrow is required. Levels of $\mathrm{HHV}-8$ replication in plasma reflect disease activity and may be useful as a follow-up parameter. Yet, this test is reserved for specialized laboratories, whereas CRP is widely available and serves, while non-specific, as a good surrogate marker for disease activity together with the clinical follow-up [5].

Epidemiologic data demonstrate a highly increased incidence of MCD in HIV-infected individuals compared to immunocompetent persons. Improvement of the immunologic response and treatment of the underlying disease is crucial, irrespective of the cause of immunosuppression. However, there is no firm evidence of a close correlation between the $\mathrm{CD} 4^{+} \mathrm{T}$-lymphocytes cell count or the use of antiretroviral therapy and the resolution of MCD. Interestingly, in a study by Powles et al. [6], the calculated incidence retrieved from the Chelsea and Westminster HIV Cohort showed an increase of MCD in the era of highly active antiretroviral therapy, while other HIV-related diseases, such as Kaposi's sarcoma, became less frequent. It is not clear whether this is a reporting bias due to a higher number of biopsies or a real increase of MCD in the population of HIV-infected individuals.

Due to the association of active HHV-8 replication with MCD, antiviral agents such as ganciclovir/valganciclovir have been successfully used [7]. Another promising approach for treatment in selected cases is rituximab, a chimeric monoclonal antibody against CD20 protein on HHV-8-infected CD20-presenting cells. Several trials have demonstrated a partial to complete remission and suppression of $\mathrm{HHV}-8$ replication after a 3-month course of treatment with rituximab [8]. Very recent data show that the introduction of rituximab-based therapy has improved the outcome dramatically [9]. Antineoplastic agents such as etoposide or vinblastine have been demonstrated to be highly active in preventing the evolution of MCD towards lymphoma [10]. However, the long-term administration of these agents is limited by possible oncogenic effects.

Because of the unspecific findings, MCD can be difficult to diagnose in the setting of episodic fever and malaise. The optimal treatment regime for MCD is not yet established and standard therapy protocols do not exist. Untreated, it leads to significant morbidity and death. We advocate exploring for MCD in unclear febrile episodes, as recent advances in treatment appear promising.

Conflict of interest None.

\section{References}

1. Castleman B, Towne VW. Case records of the Massachusetts General Hospital: case no. 40231. N Engl J Med. 1954;250: 1001-5.

2. Oksenhendler E. HIV-associated multicentric Castleman disease. Curr Opin HIV AIDS. 2009;4:16-21.

3. Waterston A, Bower M. Fifty years of multicentric Castleman's disease. Acta Oncol. 2004;43:698-704 (Review).

4. Mylona EE, Baraboutis IG, Lekakis LJ, et al. Multicentric Castleman's disease in HIV infection: a systematic review of the literature. AIDS Rev. 2008;10:25-35.

5. Oksenhendler E, Carcelain G, Aoki Y, et al. High levels of human herpesvirus 8 viral load, human interleukin-6, interleukin10 , and $\mathrm{C}$ reactive protein correlate with exacerbation of multicentric Castleman disease in HIV-infected patients. Blood. 2000; 96:2069-73.

6. Powles T, Stebbing J, Bazeos A, et al. The role of immune suppression and HHV-8 in the increasing incidence of HIVassociated multicentric Castleman's disease. Ann Oncol. 2009;20:775-9.

7. Casper C, Krantz EM, Corey L, et al. Valganciclovir for suppression of human herpesvirus- 8 replication: a randomized, double-blind, placebo-controlled, crossover trial. J Infect Dis. 2008; 198:23-30.

8. Gérard L, Bérezné A, Galicier L, et al. Prospective study of rituximab in chemotherapy-dependent human immunodeficiency virus associated multicentric Castleman's disease: ANRS 117 CastlemaB Trial. J Clin Oncol. 2007;25:3350-6.

9. Bower M, Newsom-Davis T, Naresh K, et al. Clinical features and outcome in HIV-associated multicentric Castleman's disease. J Clin Oncol. 2011;29:2481-6.

10. Scott D, Cabral L, Harrington WJ Jr. Treatment of HIV-associated multicentric Castleman's disease with oral etoposide. Am J Hematol. 2001;66:148-50. 